\title{
The 9-0 -acetyl GD3 gangliosides are expressed by migrating chains of subventricular zone neurons in vitro
}

\author{
L.M. Miyakoshi, \\ R. Mendez-O tero and \\ C. Hedin-Pereira
}

\author{
Instituto de Biofísica Carlos Chagas Filho, \\ Universidade Federal do Rio de Janeiro, \\ Rio de Janeiro, RJ, Brasil
}

\section{Correspondence \\ C. Hedin-Pereira \\ Instituto de Biofísica \\ Carlos Chagas Filho, UFRJ \\ CCS, BI. G, Cidade Universitária \\ 21941-590 Rio de Janeiro, RJ \\ Brasil \\ E-mail: hedin@ biof.ufrj.br \\ Presented at \\ SIMEC 2000 - International \\ Symposium on Extracellular \\ Matrix, Angra dos Reis, RJ, \\ Brazil, September 24-27, 2000. \\ Research supported by FINEP/ \\ PRO NEX (No. 052/97), the Third \\ W orld Academy of Science (TWAS) \\ and FAPERJ to R. Mendez-O tero.}

Received January 9, 2001

Accepted January 30, 2001

\begin{abstract}
Neurons from the anterior subventricular zone (SVZ) of the cerebral cortex migrate tangentially to become interneurons in the olfactory bulb during development and in adult rodents. This migration was defined as neuronophilic, independent of a radial glial substrate. The cortical SVZ and the rostral migratory stream to the olfactory bulb were shown to be rich in 9-O-acetyl GD3 gangliosides (9-O-acGD3), which have been previously shown to be implicated in gliophilic migration in the rodent cerebral cortex and cerebellum. In the present study, we performed SVZ explant cultures using rats during their first postnatal week to analyze the expression of these gangliosides in chain migration of neuronal precursors. We characterized migrating chains of these neuroblasts through morphological analysis and immunocytochemistry for the neural cell adhesion molecule. By using the Jones monoclonal antibody which binds specifically to 9-O-acGD3 we showed that migrating chains from the SVZ explants express 9-OacGD3 which is distributed in a punctate manner in individual cells. 9$\mathrm{O}$-acGD3 is also present in migrating chains that form in the absence of radial glia, typical of the neuronophilic chain migration of the SVZ. Our data indicate that 9-O-acetylated gangliosides may participate in neuronophilic as well as gliophilic migration.
\end{abstract}

During development of the cerebral cortex most neuronal precursors migrate radially from the ventricular zone to their final destination in the cortical plate (for a review, see 1). Radial glia-mediated migration, termed gliophilic, appears to be the prevalent form of migration in the cerebral cortex. However, long tangential migratory pathways have been described, both deriving from cortical proliferative zones to extracortical sites (for a review, see 2), as well as
Key words

- Subventricular zone

- Migration

- 9-0-Acetylated gangliosides

- Rostral migratory stream from extracortical zones to the cortical plate (3). Neurons from the anterior subventricular zone (SVZ) of the cerebral cortex migrate tangentially via the rostral migratory stream to the olfactory bulb, originating interneurons therein. In the adult, continuous neurogenesis in the SVZ provides neurons that migrate to the olfactory bulb. Lois et al. (4) have shown the ultrastructure of this pathway in adult rodents, suggesting that these neuronal precursors migrate along each other 
independent of radial glial guides, the socalled neuronophilic migration. They also showed that these chains of migrating neuroblasts are ensheathed by glial cells that form tube-like structures.

Recently, it has been possible to characterize chain migration in vitro using anterior SVZ explant cultures (5). In this model, neuronal precursors that migrate in chains out of SVZ explants express polysialic acid (PSA)-neural cell adhesion molecule (NCAM) $(6,7)$ and TuJ1 $(8)$ as was shown in vivo. These chains were also shown not to have glial fibrillary acidic protein (GFAP)labeled processes growing into them, further characterizing this migration as neuronophilic. Little is known of the molecular mechanisms that underlie chain migration. There is evidence that enzymatic removal of polysialic acid chains from NCAM (9) or the genetic deletion of PSA-NCAM disrupts neuronal precursor migration in vivo (10-12). The in vitro analysis of SVZ migration in adults showed that chain migration still occurs in transgenic mice in which all isoforms of NCAM are deleted (13).

The cortical SVZ and the rostral migratory stream were shown to have a strong expression of 9-O-acetylated gangliosides during development and in adult rats (14). This sialic acid-containing glycolipid was previously associated with gliophilic migration in the cerebral cortex (15) and cerebellum (16). In the present study we analyzed the expression of 9-O-acetylated gangliosides in anterior SVZ explant cultures to determine whether these gangliosides could be involved in neuronophilic migration. To this end we studied migrating chains of neuronal precursors in which glial cells were absent.

We prepared anterior SVZ explants from postnatal day P0-P7 rat brains. Animals were decapitated, their brains were removed and placed in cool Gey's solution and cut parasagittally at 350-400 $\mu \mathrm{m}$ with a tissue chopper (McIlwain, Gomshall, Surrey, England).
From these slices we dissected out the most anterior portion of the SVZ to obtain our explants. Explants were plated onto glass coverslips previously incubated with polyL-lysine. Our cultures were fed with culture medium (10\% fetal calf serum and DMEMF12-Gibco, Gaithersburg, MD, USA) and kept in an incubator $\left(5 \% / 95 \% \mathrm{CO}_{2} /\right.$ air $)$ for 4-7 days.

Explant cultures were fixed with $4 \%$ paraformaldehyde and submitted to immunocytochemistry. The explant cultures were incubated overnight with Jones monoclonal IgM (1:200, Sigma Chemical Co., St. Louis, MO, USA), anti-GFAP polyclonal IgG (1:400, Dako, Carpinteria, CA, USA) and antiNCAM monoclonal IgG (1:10, Sigma) solutions prepared with $5 \%$ normal goat serum, $0.05 \%$ Triton X-100 (except for Jones mAb) in PBS. After this period, cultures were washed and incubated for $2 \mathrm{~h}$ with secondary antibodies linked to $\mathrm{Cy} 3$ (IgM specific and whole molecule, 1:1000), FITC (1:100; Accurate, Westbury, NY, USA) and FITC (IgG specific, 1:50; Cappel, Aurora, OH, USA) prepared in the same solutions as above. Cultures were analyzed for the presence of migrating chains of neurons using a Zeiss inverted fluorescence microscope equipped with rhodamine, FITC and UV filters in addition to differential interferential contrast (DIC) and phase contrast microscopy. Images were captured with a Kodak digital camera (MDS 120) or with a Zeiss confocal microscope with 480 and $543 \mathrm{~nm}$ lasers.

We first identified the presence of neuronal migrating chains using DIC or phase contrast microscopy in which previously described typical morphological features could be identified (5). Migrating cells were closely apposed in the chains (Figure 1A,B) but bipolar migrating cell profiles could be identified within the bundle. Ramified extensions similar to growth cone filopodia extended from the migrating cells, as previously described (5). To further characterize 
these cells as SVZ neuronal precursors, explants were immunoreacted for NCAM. All migratory chains that were identified as SVZ chains in DIC were also immunolabeled for NCAM. It should be noted, however, that this antibody is not entirely specific for the embryonic isoform of NCAM that carries the PSA sequence. In our culture system thick migrating chains grew out of the explants - several cell bodies wide and also 3 to 5 cell bodies high as can be demonstrated by depth analysis (Zeiss LSM version 3.98, copyright 1997) of 3D-reconstructed confocal images of NCAM-positive neurons. (Figure 1C). Furthermore, previous work has shown that different postnatal brain regions close to the SVZ trajectory such as the overlying cortex, striatum, medial septum, hippocampus, hypothalamus and habenula do not form chains in explant cultures (5).

Typical migrating chains out of SVZ explants expressed the ganglioside 9-O-acetyl GD3 in most cells and generally displayed a punctate distribution within cell membranes (Figure 2). This ganglioside exhibits a similar punctate pattern of distribution in growth cones of dorsal root ganglion neurons in culture (17). This distribution may be related to adhesion sites necessary for cell and growth cone translocation. To study if the migration of ganglioside-positive neuronal precursors occurred independent of glial cells we ana-
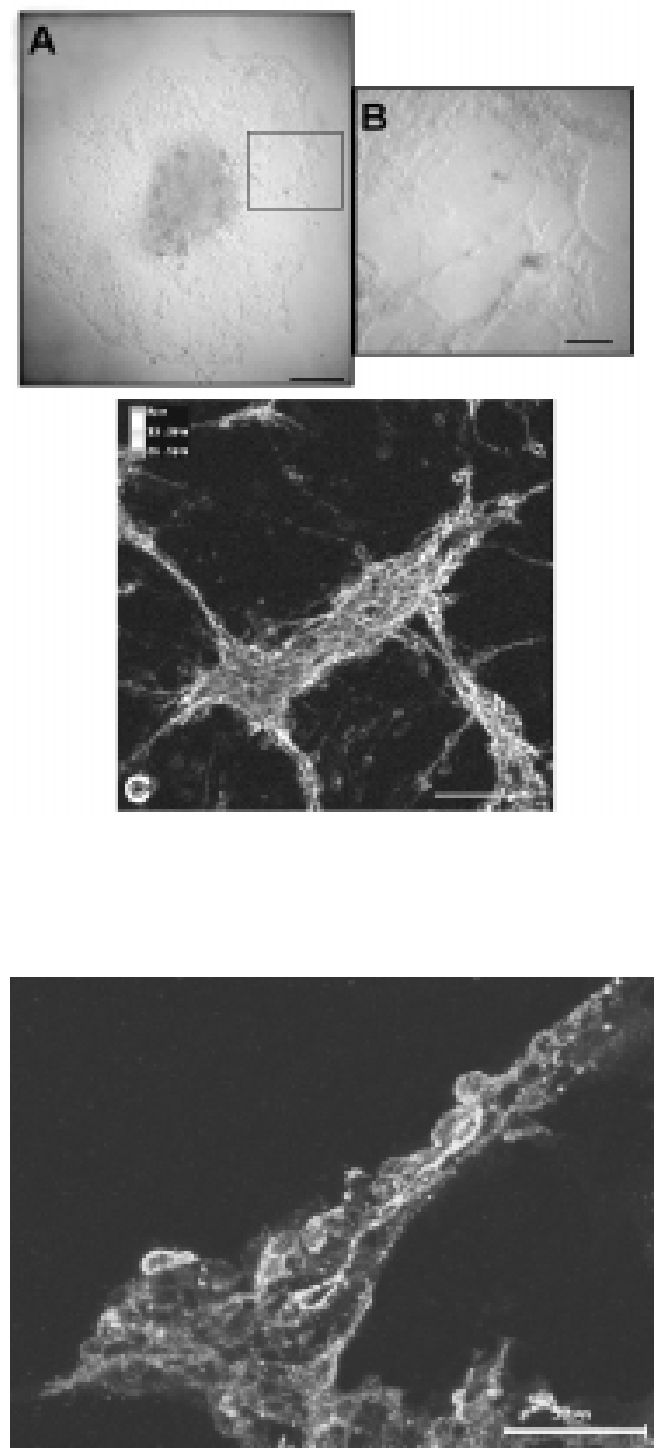

Figure 2. Confocal microscopy of J ones-positive neurons in migrating chains of neuroblasts. Note the punctate pattern of the membrane labeling. Magnification bar: $50 \mu \mathrm{m}$.
Figure 1. Immunolabeling for NCAM in subventricular zone explants. A, DIC microscopy image of a subventricular zone explant presenting migratory chains of neuroblasts. These chains can be viewed at high power in B. C, Confocal microscopy image of a migratory chain immunolabeled for NCAM viewed in a depth code image showing that these chains are organized as thick bundles of neuroblasts. Magnification bars in A: $200 \mu \mathrm{m}, \mathrm{B}: 100 \mu \mathrm{m}$, and C: $50 \mu \mathrm{m}$.
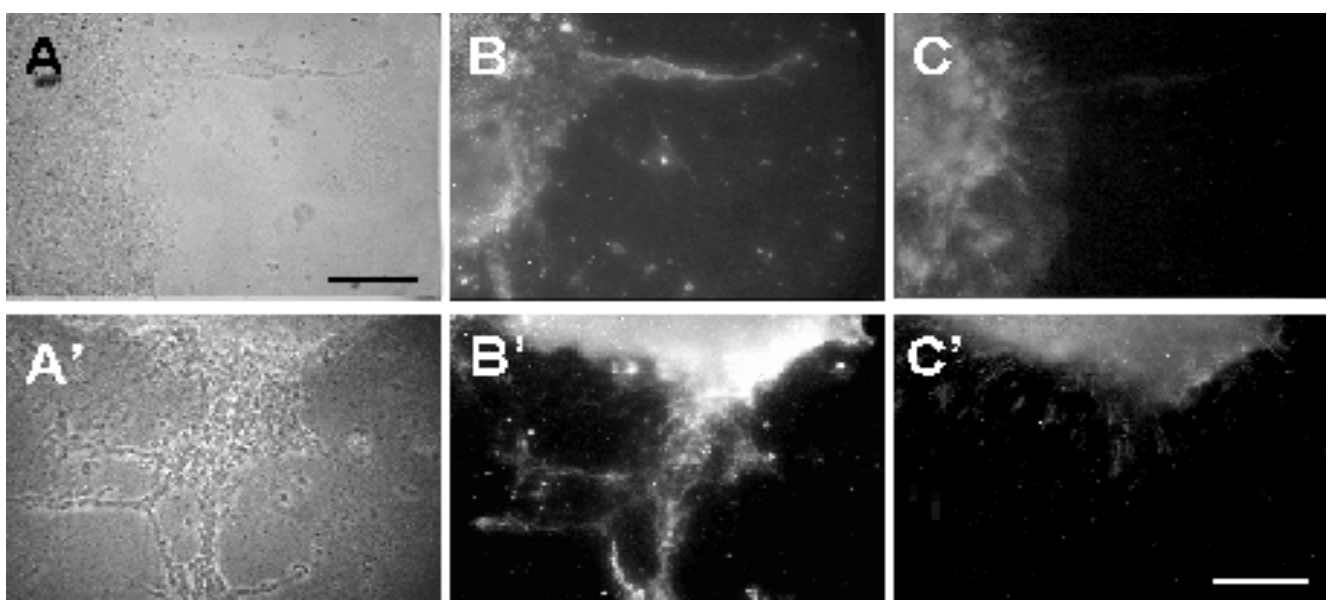

Figure 3. Migrating chains of neuroblasts from subventricular zone explants. A and $A^{\prime}$, DIC and phase contrast microscopy images. B and B', Immunolabeling for J ones showing that the chains of neuroblasts express 9 O-acetyl GD3 ganglioside. $C$ and $C^{\prime}$, Immunolabeling for GFAP, showing that these chains are able to migrate in the absence of glial fibers. Magnification bar: $200 \mu \mathrm{m}$. 
lyzed the expression of GFAP in these cultures. Typically, GFAP-positive fibers extended out of the SVZ explants (Figure 3). Differentiated astrocytes can be identified underlying some of the growing chains. However, migrating chains present in the periphery of the explant outgrowth appeared to be completely devoid of GFAP-positive processes (Figure 3). When double-labeling cultures with anti-GFAP and Jones we found that neuronal precursor chains in which glial processes were absent expressed the Jones antibody (Figure 3 ). These results suggest that the 9-O-acetyl GD3 ganglioside may participate in chain migration, which has been shown to be independent of glial guidance. Previous work has strongly associated these sialogangliosides with gliophilic migration (for a review, see 18). They are expressed in most proliferative zones and along radial glial fibers in a spatiotemporal pattern consistent with its role in promoting migration (19). In mixed cultures of cerebellar neurons and glia, elongated glial forms appear which are able to support migration. It has been shown in this system that these glial cells only express the ganglioside when serving as substrate for neuronal migration (20). In migration assays using cerebellar explants, immunoblockade of the 9-O-acetyl GD3 ganglioside disrupts neuronal precursor migration along radial glial fibers (16). Our results substantiate the hypothesis that 9-O-acetylated gangliosides participate in neuronophilic migration. The mechanism underlying this action remains to be determined.

\section{Acknowledgments}

We are grateful to José Tibúrcio for animal care and to Felipe de Melo Marins for technical assistance.

\section{References}

1. Rakic P (1990). Principles of neural migration. Experientia, 46: 882-891.

2. Goldman SA \& Luskin MB (1998). Strategies utilized by migrating neurons of the postnatal vertebrate forebrain. Trends in Neurosciences, 21: 107-114.

3. Anderson SA, Eisenstat DD, Shi $L \&$ Rubenstein J LR (1997). Interneuron migration from basal forebrain to neocortex: dependence on DIx genes. Science, 278: 474-476.

4. Lois C, Garcia-Verdugo J M \& AlvarezBuylla A (1996). Chain migration of neuronal precursors. Science, 271: 978-981.

5. Wichterle H, García-Verdugo J M \& AlvarezBuylla A (1997). Direct evidence for homotypic, glia-independent neuronal migration. Neuron, 18: 779-791.

6. Rousselot $P$, Louis $C \&$ Alvarez-Buylla $A$ (1995). Embryonic (PSA) N-CAM reveals chains of migrating neuroblasts between the lateral ventricle and the olfactory bulb of adult mice. J ournal of Comparative Neurology, 351: 51-61.

7. J ankovski A \& Sotelo C (1996). Subventricular zone-olfactory bulb migratory pathway in the adult mouse: Cellular composition and specificity as determined by heterochronic and heterotopic transplantation. J ournal of Comparative Neurology, 371: 376-396.

8. Menezes JRL \& Luskin MB (1994). Expression of neuron-specific tubulin defines a novel population in the proliferative layers of the developing telencephaIon. J ournal of Neuroscience, 14: 53995416.

9. Ono $\mathrm{K}$, Tomasiewicz $\mathrm{H}$, Magnuson $\mathrm{T} \&$ \& Rutishauser U (1994). N-CAM mutation inhibits tangential neuronal migration and is phenocopied by enzymatic removal of polysialic acid. Neuron, 13: 595-609.

10. Tomasiewicz H, Ono K, Yee D, Thompson C, Goridis C, Rutishauser $U \&$ Magnuson $T$ (1993). Genetic deletion of a neural cell adhesion molecule variant (NCAM 180) produces distinct defects in the central nervous system. Neuron, 11: 1163-1174.

11. Cremer H, Lange R, Christoph A, Plomann M, Vopper G, Roes J , Brown R, Baldwin $S$, Kraemer $P$, Scheff $S$, Barthels $D$, Rajewsky K \& Wille W (1994). Inactivation of the N-CAM gene in mice results in size reduction of the olfactory bulb and deficits in spatial learning. Nature, 367: 455459.
12. Hu HY, Tomasiewicz H, Magnuson $\mathrm{T} \&$ Rutishauser U (1996). The role of polysialic acid in migration of olfactory bulb interneuron precursors in the subventricular zone. Neuron, 16: 735-743.

13. Chazal $G$, Durbec $P$, J ankovski A, Rougon $\mathrm{G} \&$ Cremer H (2000). Consequences of neural cell adhesion molecule deficiency on cell migration in the rostral migratory stream of the mouse. J ournal of Neuroscience, 20: 1446-1457.

14. Mendez-Otero R \& Cavalcante LA (1996). Expression of 9-O acetylated gangliosides is correlated with tangential cell migration in the rat brain. Neuroscience Letters, 204: 97-100.

15. Hedin-Pereira $C$, de Moraes ECP, Lent $R$ $\&$ Mendez-Otero R (1998). 9-O Acetylated gangliosides are required for radial cell migration in embryonic cerebral cortex. Axon Guidance and Neural Plasticity Meeting. September 16-20, Cold Spring Harbor, NY, 75.

16. Santiago MF, Berredo-Pinho $M$, Costa MR, Gandra M, Cavalcante LA \& MendezOtero R (2001). Expression and function of 9-0-acetyl GD3 in postmitotic granule cell development. Molecular and Cellular 
Neurosciences (in press).

17. Araújo $\mathrm{H}$, Menezes $\mathrm{M} \&$ \& Mendez-Otero $\mathrm{R}$ (1997). Blockage of 9-O-acetylated gangliosides induces microtubule polymerization in growth cones and neurites. European J oumal of Cell Biology, 72: 202-213.

18. Mendez-Otero $\mathrm{R}$, Schlosshauer $\mathrm{B} \&$ Constantine-Paton M (1992). Role of acetylated gangliosides on neuronal migration and axonal outgrowth. In: Lent R (Editor), The Visual System from Genesis to Maturity. Birkhauser, Boston.

19. Mendez-Otero R, Schlosshauer B, Barnstable CJ \& Constantine-Paton M (1988). A developmentally regulated antigen associated with neural cell and process mi- gration. J ournal of Neuroscience, 8: 564579.

20. Mendez-Otero $\mathrm{R} \&$ Constantine-Paton $\mathrm{M}$ (1990). Granule cell induction of 9-O acetyl gangliosides on cerebellar glia in microcultures. Developmental Biology, 138: 400-409. 